\section{Commentary: Soft prognosticators following radical treatment of lung cancer: The time has come for a more integrated approach}

\author{
Alessandro Brunelli, MD
}

The study from Park and colleagues ${ }^{1}$ is an interesting analysis reporting on the inverse association between prognostic nutritional index (PNI) and incidence of postoperative adverse events and late mortality after curative resection for lung cancer. This contribution adds to the growing number of studies reporting an association between baseline patient-related characteristics (functional, metabolic, psychological) and cancer progression after curative lung resection.

As honestly stated by the authors, it is impossible from this analysis to demonstrate a real causal association between low PNI and increased rate of adverse events. Low PNI may be in fact a surrogate for other unknown or unmeasured factors more closely related with prognosis or represent an epiphenomenon caused by the more aggressive biology of the tumor.

Another limitation of this study is the lack of stratification by cancer stage. Finding the same inverse relationship between PNI and survival in early-stage patients (ie, pT1N0) would have corroborated the intriguing hypothesis of a direct (and potentially modifiable) effect on the immune system.

Finally, surgery may have a profound impact on the immunologic and inflammatory status of the patient. How this may alter the baseline PNI is unknown but can potentially affect prognosis. Perioperative changes of PNI rather than baseline values may be more closely related (directly or indirectly) to cancer evolution following treatment. This may have important implications, as it would identify the most appropriate period to target when planning to

\footnotetext{
From the Department of Thoracic Surgery, St James's University Hospital, Leeds Teaching Hospital NHS Trust, Leeds, United Kingdom.

Disclosures: Author has nothing to disclose with regard to commercial support.

Received for publication Dec 1, 2019; revisions received Dec 1, 2019; accepted for publication Dec 2, 2019; available ahead of print Dec 14, 2019.

Address for reprints: Alessandro Brunelli, MD, Department of Thoracic Surgery, St James's University Hospital, Bexley Wing, Beckett St, Leeds, LS9 7TF, United Kingdom (E-mail: alexit_2000@yahoo.com).

J Thorac Cardiovasc Surg 2020;160:287-8

$0022-5223 / \$ 36.00$

Copyright (c) 2019 by The American Association for Thoracic Surgery

https://doi.org/10.1016/j.jtcvs.2019.12.004
}

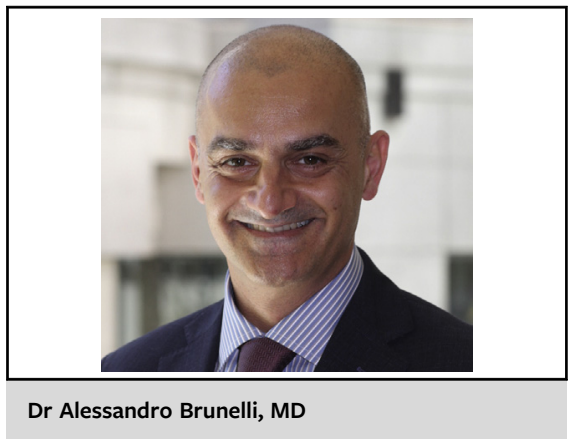

CENTRAL MESSAGE

TNM staging system should be integrated by cancer- and patient-related biologic, immunologic, and metabolic characteristics to allow a targeted and personalized cancer treatment.

implement supportive measures aimed at improving the inflammatory, immune, and metabolic status of the patient.

Similarly, patients analyzed in this study were all operated through thoracotomy. Whether these results are generalizable to patients undergoing minimally invasive surgery should be tested, as the latter approach has been shown to determine less immunologic and inflammatory derangements compared with open surgery.

The findings reported in this study ${ }^{1}$ are in line with previous investigations showing, for instance, an inverse relationship between body mass index and survival following lung resection. Low body mass index represents a surrogate marker for frailty and possibly sarcopenia, which is a known negative prognostic factor. In contrast, low albumin level and loss of muscle mass may also be the effect of a more-advanced and aggressive cancer biology.

Other studies have found a positive association between fitness and long-term survival. Better physical functioning has been shown to have beneficial effects on the immune system and body metabolism that can potentially affect cancer progression and survival.

It is becoming evident that the categorization of cancer into stages according to the traditional Tumor, Node, Metastasis (TNM) staging system is too simplistic and does not accurately capture the nuances associated with cancer progression. Cancer genetic and immune profiling and host-related factors need to be integrated into a more complex system assisting physicians in targeting and tailoring cancer treatment. 
The authors should be commended for exploring the relation between prognosis and less-conventional prognostic factors, highlighting the importance of a more holistic approach to cancer management.

\section{Reference}

1. Park S, Ahn HJ, Yang M, Kim JA, Kim JK, Park SJ. The prognostic nutritional index and postoperative complications after curative lung cancer resection: a retrospective cohort study. J Thorac Cardiovasc Surg. 2020; 160:276-85.e2. 